\title{
Heuristic Rules Embedded Genetic Algorithm to Solve In-Core Fuel Management Optimization Problem
}

\author{
Fatih Alim \\ Mechanical and Nuclear Engineering \\ Pennsylvania State University \\ 18148808955 \\ fxa130@psu.edu
}

\author{
Kostadin Ivanov \\ Mechanical and Nuclear Engineering \\ Pennsylvania State University \\ 18148650040 \\ kni1@psu.edu
}

\begin{abstract}
Because of the large number of possible combinations for the fuel assembly loading in the core, the design of the loading pattern (LP) is a complex optimization problem. It requires finding an optimal fuel arrangement in order to achieve maximum cycle length while satisfying the safety constraints. The objective of this study is to develop a loading pattern optimization code. Generally in-core fuel management codes are written for specific cores and limited fuel inventory. One of the goals of this study is to develop a loading pattern optimization code, which is applicable for all types of Pressurized Water Reactor (PWR) core structures with unlimited number of fuel assembly types in the inventory. To reach this goal an innovative genetic algorithm is developed with modifying the classical representation of the genotype. To obtain the best result in a shorter time not only the representation is changed but also the algorithm is changed to use in-core fuel management heuristics rules. The improved GA code was tested demonstrating the advantages of the introduced enhancements. The core physics code used in this research is Moby-Dick, which was developed to analyze the VVER reactors by SKODA Inc.
\end{abstract}

\section{Categories and Subject Descriptors}

J.2 [Physical Sciences and Engineering]: - engineering.

\section{General Terms}

Algorithms, Design.

\section{Keywords}

In-core Fuel Management, Pressurized Water Reactor (PWR), Loading Pattern, Heuristic Rules, Genetic Algorithms.

\section{INTRODUCTION}

In-core fuel management optimization is one of the most important aspects of the operation of nuclear reactors. It involves the arrangement of approximately 150 to 200 fuel assemblies in the pressurized water reactor (PWR). A typical $1 / 8$ core sector of symmetry can have $10^{26}$ and more possible loading patterns. Loading pattern (LP) includes used fuel assemblies coming from previous cycles and fresh fuel assemblies which replace the

Copyright is held by the author/owner(s).

GECCO'05, June 25-29, 2005, Washington, DC, USA.

ACM 1-59593-010-8/05/0006. discharged fuel assemblies at the end of the cycle (EOC). All fuel assemblies are reshuffled to a configuration that is optimal with respect to some performance criterion and which meets the safety constraints. Usually this requires finding an optimal fuel arrangement with maximum cycle length in the reactor core while satisfying the safety constraints.

The objective of this study is to develop a loading pattern optimization code. Generally in-core fuel management codes are written for specific cores and limited fuel inventory. One of the goals of this study is to write a loading pattern optimization code, which is applicable for all types of PWR core structures with unlimited fuel assembly types in the inventory. To reach this goal an innovative genetic algorithm is developed with changing the classical representation of the genotype. To obtain the best result in a shorter time not only the representation is changed but also the algorithm is changed to use in-core fuel management heuristics rules.

The core physics code used in this study is Moby-Dick, which was developed to analyze the VVER reactors by SKODA Inc. Moby-Dick is based on the finite difference approximation to the few-group (2 -10 energy groups) diffusion equation.

\section{Using In-Core Fuel Management Heuristic Rules}

Nuclear reactors have been operated for the last 40 years. Many heuristic rules have been learned within these years to arrange the fuel assembly types in the nuclear reactor core. To use these rules for creating initial population and utilizing these rules with GA operators, worth definition application is developed. If fuel management heuristic rules state that:

- Fuel assembly type A should not be in the location x.

$$
0.0 \leq \text { Worth }_{x}^{A}<0.5
$$

- Fuel assembly type A should be in the location $\mathrm{x}$.

$$
0.5<\text { Worth }_{x}^{A} \leq 1.0
$$

- There is no idea for the location of fuel assembly type A for location $\mathrm{x}$.

$$
\operatorname{Worth}_{x}^{A}=0.5
$$


For creation of initial population a subroutine provides a percent of individuals, which have genes with higher than 0.5 worth. This number can be defined by the user. To provide diversity in the population $20 \%$ of population's worth values can be lower than 0.5 .

\section{Age Process}

To use heuristic rules effectively and decrease the problem size, age process is developed. Operation time is divided to ages as the ages in the real world life. (For example ice age). It is assumed that in each age operators works with a group of genes instead with all of the genes. Groups are created according to the in-core fuel management heuristic rules and obtained frequency values in the first run. Frequency values define the frequency of each fuel assembly type loading in each location for the better LPs.

\section{Results}

The comparison of the classical GA with the heuristic rules embedded GA is shown in Figure 1. In this figure the ages are divided with line. The obtained results show that the heuristic rules embedded GA gives better fitness value in a shorter time as compared to the classical GA.

Genotype with higher fitness defines LP with longer cycle length and maximum NP lower than 1.3. Figure 1 shows that the heuristic rules embedded GA finds much better LP at the beginning of the run and this LP is very close to the LP, which is found by the classical GA at the $250^{\text {th }}$ generation. While the heuristic rules embedded GA finds the optimal LP (fitness is $3.602)$ at $176^{\text {th }}$ generation, the classical GA finds a LP with lower fitness (fitness is 3.587 ) at $250^{\text {th }}$ generation.

\section{Conclusion}

The genetic algorithm was used to optimize LP elsewhere. In this research, The GA concept for LP problem optimization is adjusted further by using some novel developments. These developments are summarized below;

- The previous in-core fuel management codes are written for specific core structures with using different techniques such as deterministic and stochastic methods. This research is aimed to developing a general code, which is suitable for every core structure. GA is suitable for this goal. A new genotype representation is developed to define the different core structures by specifying in the input deck easily. Then, GA operators are modified to adapt the new representation and are applied to generate new populations.

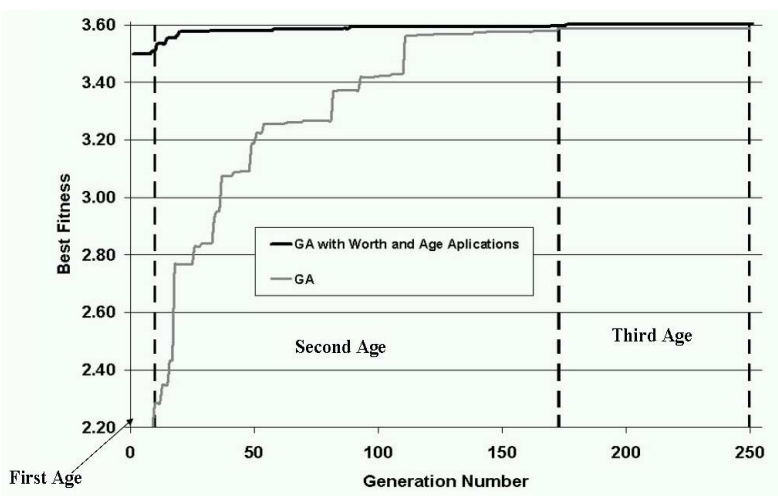

Figure 1. Comparison of the classical GA with the heuristic rules embedded GA

- In the last 50 years, the experience of operation of nuclear reactors provided in-core fuel management heuristic rules for loading pattern optimization. Using these rules in the optimization code will decrease the time to obtain optimal results. To use these rules, the worth definition concept is developed and combined with the GA code. Population is created with using worth definition and some restrictions are added to the operators to use worth definitions to create next generations. When using this concept the running time of the code is decreased.

- The in-core re-load problem is very large problem. It includes approximately $10^{26}$ different combinations. If the burnable poison optimization is added to the problem, the size of problem will be larger than $10^{26}$. The size of the problem is decreased during the operation of the GA code with using the age definition, which is combined with the fuel management heuristic rules.

\section{References}

[1] Alim F., and Ivanov, K., Genetic Algorithm Development for in-Core Fuel Management, ANS 2004 Annual Meeting, June 13-17 2004.

[2] Alim F., and Ivanov, K., Modeling Genetic Algorithm Operators for Loading Pattern Optimization, ANS 2004 Winter Meeting, November 14-18 2004

[3] DeChaine, M., and Feltus, M., Stochastic Fuel Management Optimization Using Genetic Algorithms and Heuristic Rules, Ph.D. Thesis in Nuclear Engineering, the Pennsylvania State University, 1995 